\title{
Von Zustand und Zukunft des Westens - Ein Bericht zur 56. Münchner Sicherheitskonferenz
}

\author{
Randolf Carr $\cdot$ Christoph Erber
}

Online publiziert: 17. Juni 2020

(C) Springer Fachmedien Wiesbaden GmbH, ein Teil von Springer Nature 2020

\section{Eine westliche „Identitätskrise“?}

„Die Welt ist ein ziemlich gefährlicher und oft gesetzloser Ort geworden. Und währenddessen scheint der Westen eine Art Identitätskrise zu durchleben." Münchner Sicherheitskonferenz (Munich Security Conference, MSC), die oft verkürzt als ein Familientreffen westlicher Verbündeter bezeichnet wird, begann am 14. Februar 2020 mit diesen Worten ihres Vorsitzenden, Botschafter Wolfgang Ischinger. Damit wurde klar, dass dieses Jahr statt eines „Requiems für den Westen“ (Cohen 2019, eigene Übersetzung), wie die Konferenz des Vorjahres teilweise zusammengefasst wurde, die Forderung nach Antworten auf die Krise der westlichen Wertegemeinschaft im Mittelpunkt stehen würde. Für das weitverbreitete Unbehagen angesichts einer zunehmenden Infragestellung westlicher Werte, die rund um die Welt sowie auch innerhalb westlicher Gesellschaften stattfindet, hatte bereits die Begleitpublikation der Konferenz, der Munich Security Report 2020, den Neologismus „Westlessness“ geschaffen (Bunde et al. 2020). Westlessness, in der deutschen Presse häufig als „Westlosigkeit“ übersetzt, macht sich, so der Report, auch auf internationaler Ebene im Verlust des westlichen Gestaltungsanspruchs bemerkbar (Bunde et al. 2020) - mit unmittelbaren Auswirkungen auf viele drängende Sicher-

\footnotetext{
1 Soweit nicht anders vermerkt, basieren die Zitate der Konferenz auf den veröffentlichten Redetexten oder auf eigener Transkription und/oder Übersetzung. Mitschnitte der Reden und Panel-Debatten finden Sie auf der Internetseite der Münchner Sicherheitskonferenz: https://www.securityconference.org/en/medialibrary/munich-security-conference-2020/.
}

R. Carr · C. Erber $(\bowtie)$

Münchner Sicherheitskonferenz gGmbH, Karolinenplatz 3, 80333 München, Deutschland

E-Mail: erber@securityconference.org

R. Carr

E-Mail: carr@securityconference.org 
heitsherausforderungen. Über diese und über den Zustand des Westens wurde in München während des Hauptprogramms ${ }^{2}$ und bei mehr als 200 Begleitveranstaltungen und 2000 bilateralen Treffen gesprochen. Über 30 Staats- und Regierungschefs und knapp 100 Minister*innen aus aller Welt fanden sich ein, um an den Debatten teilzunehmen.

\section{Der Westen - mehr als eine Himmelsrichtung?}

Das Kunstwort Westlessness sorgte für eine kontroverse Diskussion. Für viele Beobachter*innen der Konferenz kristallisierte sich an den gegensätzlichen Auftritten des deutschen Bundespräsidenten Frank-Walter Steinmeier und des US-Außenministers Mike Pompeo die innerwestliche Uneinigkeit über die Grundlagen der Wertegemeinschaft heraus. Die USA „erteilen unter der jetzigen Regierung selbst der Idee einer internationalen Gemeinschaft eine Absage“, beklagte Steinmeier. Den Bundespräsidenten direkt zitierend entgegnete Pompeo am Folgetag, diese Behauptungen hätten ,keinen Bezug zur Realität.“ In seiner Rede zeichnete Pompeo das Bild eines Westens, der nicht in der Krise, sondern unter US-amerikanischer Führung auf dem Siegeszug sei. Allerdings teilten wenige im Publikum diese optimistische Einschätzung. So titelte The New York Times: „'The West Is Winning,' Pompeo Said. The West Wasn't Buying It“ (Sanger und Erlanger 2020). Denn trotz Pompeos Bekenntnissen zu liberalen Werten und Multilateralismus zogen viele das ernüchternde Resümee, dass die US-Regierung offenkundig „Souveränität und nicht Kooperation als oberstes Gebot" (Brössler 2020) in den internationalen Beziehungen sieht. In den kritischen Worten Steinmeiers: „Als ob an alle gedacht sei, wenn jeder an sich denkt." In Zeiten, in denen für viele Teilnehmende die Antwort auf die Krise des Westens ,mehr Europa“ lauten würde, schien das Loblied auf nationale Souveränität des US-Außenministers auf wenig Gegenliebe zu stoßen (Fontaine 2020).

Es wurde jedoch auch deutlich, dass Westlessness weit über die Grenzen des traditionellen geografischen Westens hinaus beschäftigt. Gerade für Vertreter*innen aus Asien signalisierte der Begriff eine überfällige westliche Erkenntnis über den Zustand der Welt: Nach einer Ära der westlichen Dominanz - vom indischen Außenminister Subrahmanyam Jaishankar auf der Bühne spontan als „Westfulness“ tituliert - sortiere sich die Welt nun sowohl wirtschaftlich als auch politisch neu. Die Kommissions-Vizepräsidentin der Europäischen Union Margrethe Vestager pflichtete bei, dass gerade Europa sich in dieser Weltlage neu orientieren müsse. Der Begriff des Westens sei dabei eher ein hinderlicher Anachronismus. Ob es den Westen als Synonym für eine multilaterale, liberale Wertegemeinschaft noch braucht, stellte Kang Kyung-wha, die südkoreanische Außenministerin, ebenfalls infrage: „Die Werte, die dem Multilateralismus zugrunde liegen, sind nicht mehr nur dem Westen zu eigen. Sie sind jetzt universelle Werte [...]." Die Diskussion um Westlessness in München auf die Dissonanz zwischen den USA und Europa zu reduzieren, wäre also verkürzt. Ebenso wie die Bedrohung der westlich geprägten Weltordnung nicht nur von

\footnotetext{
${ }^{2}$ Einen Überblick über die Teilnehmerliste und Agenda der diesjährigen Konferenz erhalten Sie auf der Internetseite der Münchner Sicherheitskonferenz: https://securityconference.org/msc-2020/agenda.
} 
außerhalb des Westens kommt - die ideologischen Trennlinien verlaufen vielmehr quer durch die Gesellschaften (Bunde, zit. n. hr2-kultur 2020) -, so werden ihre grundlegenden Werte in anderen Weltregionen gelebt und verteidigt.

\section{Westliche Handlungsfähigkeit schmerzlich vermisst}

Während der Westen seine Gestaltungsmacht zunehmend einbüßt, steht er, wie Ischinger mahnte, vor einem ,unerträglichen Zustand globaler Unsicherheit“. Viele Beobachter*innen beeindruckte vor allem, wie das Thema „China, China, China“ (Casdorff 2020) die Diskussionen auf der Konferenz prägte. Bezeichnend war die Rede des US-Verteidigungsministers Mark Esper, der für das europäische Publikum die Vorteile US-amerikanischer „Führungsstärke“ und die Gefahren chinesischer „Heimtücke“ ausbuchstabierte. Es regten sich Hoffnungen, dass die Konferenz zur transatlantischen Annäherung in Bereichen wie der 5G-Debatte beitragen könnte (Drezner 2020). Doch viel mehr wurden die Schwierigkeiten der USA deutlich, einen Schulterschluss mit Europa gegenüber China zu erwirken. China und Russland, die erklärten Konkurrenten der liberalen Wertegemeinschaft, schaffen dagegen weiter Fakten und übernehmen stückweise die Deutungshoheit über Themen, die Kerninteressen des Westens betreffen. So etwa im Nahen Osten, dessen Zukunft wie der deutsche Außenminister Heiko Maas beklagte - zunehmend ,in Astana oder Sotschi entschieden [wird], anstatt in Genf oder New York." In den Diskussionen zu globalen Bedrohungen wie dem Klimawandel und der Coronavirus-Krise wurde zwar eindringlich zu internationaler Geschlossenheit aufgerufen - doch auch bei diesen beiden Themen wurden letztlich die Defizite in der westlichen Einigkeit und gemeinsamen Handlungsfähigkeit offenkundig.

\section{Miteinander statt übereinander reden}

Inmitten dieser Herausforderungen untermauerte die MSC 2020 aber auch ihren Status als führende Dialogplattform für sicherheitspolitische Fragen und zwischenstaatliche Konflikte. So diskutierten der Premierminister Armeniens Nikol Paschinjan und der Präsident Aserbaidschans Ilcham Alijew erstmals öffentlich über den Konflikt um die Region Bergkarabach, der die beiden Länder seit knapp 30 Jahren entzweit. Allerdings hielten sich die beiden Regierungschefs noch zu sehr an historischen (Schuld-)Fragen auf, was Fortschritte in den gegenwärtigen Verhandlungen erschwert (De Waal 2020). Von einer langen Vorgeschichte geprägt ist auch der ungelöste israelisch-palästinensische Konflikt, der jüngst durch einen umstrittenen Lösungsvorschlag von US-Präsident Donald Trump neue Aufmerksamkeit erhielt. Jener Nahostkonflikt war Thema in einem der zehn interaktiven TownhallFormate. Unter reger Beteiligung eines hochrangigen Publikums entwickelte sich eine leidenschaftliche, aber respektvolle Diskussion mit dem Ministerpräsidenten der Palästinensischen Autonomiegebiete Mohammed Schtajjeh, dem Außenminister Jordaniens Ayman Safadi und dem Generalsekretär der Arabischen Liga Ahmed 
Aboul Gheit. Aus dem voll besetzten Saal kamen Stimmen aus den USA, Israel, Europa und der arabischen Welt zu Wort.

Gleichzeitig war die MSC 2020 auch Treffpunkt vieler multilateraler Initiativen. Auf Einladung der deutschen Verteidigungsministerin Annegret Kramp-Karrenbauer und ihres US-amerikanischen Amtskollegen Esper trafen sich Vertreter*innen der großen truppenstellenden Staaten der Anti-IS-Koalition und plädierten geschlossen für eine Fortsetzung des gemeinsamen Einsatzes gegen die Extremistenmiliz. „Wir haben große Fortschritte erzielt“, aber der „Kampf gegen den IS ist nicht beendet", so Kramp-Karrenbauer (zit. n. BMVg 2020). Daneben lud ihr Kabinettskollege Maas die Teilnehmerstaaten der Berliner Libyen-Konferenz ein, um weitere Herausforderungen des Konflikts zu beraten - insbesondere das bis dato weitestgehend unwirksame Waffenembargo der Vereinten Nationen gegen Libyen. Der Hohe Vertreter der EU für Außen- und Sicherheitspolitik Josep Borrell Fontelles forderte daher ein entschiedeneres europäisches Vorgehen, um den Konflikt zu befrieden. Die EU solle ,in der Lage sein zu handeln [...] und nicht jeden Tag Kommentare abgeben, in denen wir unsere Sorge ausdrücken“ (Borrell Fontelles, zit. n. zdf.de 2020).

\section{Europa zwischen Macht und Machbarkeit}

Für größere europäische Handlungsfähigkeit warb auf der MSC 2020 neben dem EU-Außenbeauftragten auch der französische Präsident Emmanuel Macron. Im Gespräch mit Ischinger und dem Publikum in München forderte er, dass Europa in außenpolitischen Fragen mehr mit einer Stimme sprechen müsse - und wurde damit in den Augen vieler zum „Star“ der Konferenz (Joffe 2020). Wie eine europäische „Sprache der Macht“ (Borrell Fontelles 2019, eigene Übersetzung) zukünftig klingen könnte, wird sich vor allem in den Dimensionen Wirtschaft, Technologie und Militär entscheiden, deren Potential (noch) nicht vollständig ausgeschöpft wird.

Das ökonomische Gewicht der EU - immerhin ein Sechstel des globalen Volkseinkommens - lässt sich jedoch nicht einfach in geopolitischen Einfluss umwandeln. Tatsächlich versucht Europa dies mittels 42 laufender Sanktionsprogramme bereits umfangreich (Russell 2018). Allerdings zeigt das bereits erwähnte Beispiel des Libyen-Konfliktes auch: Sofern die EU nicht glaubwürdig mit wirtschaftlichen und finanziellen Sanktionen drohen kann - etwa aufgrund mangelnder Einigkeit zwischen ihren Mitgliedstaaten -, nützt ihre wirtschaftliche Stärke wenig, um Verstöße gegen internationales Recht zu ahnden (Kaim und Schulz 2020). Die Prinzipien des multilateralen Handelssystems setzen der EU ebenfalls Grenzen für den geopolitischen Einsatz der eigenen Handelspolitik, erläuterte beispielsweise die geschäftsführende Direktorin des Internationalen Währungsfonds Kristalina Georgieva auf der MSC 2020. Damit ist fraglich, ob der von der EU im letzten Jahr modernisierte Werkzeugkasten an Handelsschutzinstrumenten den Anspruch einer europäischen Gestaltungsmacht untermauern kann.

Dass Europa auch im technologischen Wettbewerb hinter seinen Möglichkeiten zurückbleibt, war ebenfalls ein Kernthema in München. Europa sei hier auf dem Weg, ein „Kontinent zu werden, der nicht an seine Zukunft glaubt“, so Macron. 
Dabei verfügt Europa, wie in der 5G-Debatte rund um die Konferenz vielfach hervorgehoben wurde, mit Nokia und Ericsson über zwei führende Unternehmen in diesem strategischen Bereich. In vielen Diskussionen abseits der Hauptbühne kursierte daher der Vorschlag, mit den beiden Mobilfunk-Ausrüstern ein europäisches Technologie-Konsortium - nach dem Vorbild von Airbus - zu schaffen. Von deutscher Seite griff etwa die Bundesvorsitzende der Grünen Annalena Baerbock den Vorschlag auf und deutete an, dass Europa im Umgang mit kritischen Zukunftstechnologien noch viel größere Herausforderungen bevorstehen (Rinke 2020). Die Einsicht, dass die gegenwärtige 5G-Diskussion dafür also allemal nur ein Vorbote ist, wird für die europäische Konkurrenz- und Handlungsfähigkeit entscheidend sein.

$\mathrm{Zu}$ europäischer Handlungsfähigkeit zählt auch eine engere Zusammenarbeit in Verteidigungsfragen. Viele der europäischen Teilnehmenden waren sich darin einig, dass ein starkes Bündnis mit den USA für die Sicherheit Europas weiter unverzichtbar sei - aber es mangelte auch nicht an Ideen, wie Europa angesichts transatlantischer Unsicherheit seine konventionelle und nukleare Verteidigungsfähigkeit sicherstellen kann. Für Diskussionen hatte im Vorfeld der Konferenz Präsident Macron gesorgt, der Anfang Februar in einer Grundsatzrede den europäischen Partnern einen strategischen Dialog zur nuklearen Abschreckung angeboten hatte (Présidence de la République 2020). In diesen und anderen Verteidigungsfragen müsse das deutschfranzösische Tandem eine europäische Perspektive vorantreiben - alles andere sei „ein historischer Fehler.“ Der französische Präsident bekannte jedoch, dass er zunehmend ,ungeduldig“ auf Antworten aus Berlin warte.

Tatsächlich waren die Beiträge von Vertreter*innen der Bundesrepublik versucht, einer handlungsorientierteren deutschen Sicherheitspolitik ein Gesicht zu verleihen. Allerdings bemängelten Expert*innen eine ,fehlende Positionierung Deutschlands“ in einigen entscheidenden Zukunftsfragen (Bialecki et al. 2020). Viele im Publikum mögen sich dabei an den Münchner Konsens der Sicherheitskonferenz 2014 erinnert gefühlt haben. Damals hatten Bundespräsident Joachim Gauck, der damalige AuBenminister Frank-Walter Steinmeier und Verteidigungsministerin Ursula von der Leyen einstimmig ,mehr deutsche Verantwortung“ gefordert (Bunde 2014). Sechs Jahre später konstatierte von der Leyens Nachfolgerin Kramp-Karrenbauer, dass diesem „Münchner Konsens der Worte“ nun außenpolitische Taten folgen müssen. Für Deutschland sei die enge Zusammenarbeit mit seinen europäischen Nachbarn dabei der ,unabdingbare Rahmen für unsere Selbstbehauptung in der Welt“, erklärte Bundespräsident Steinmeier. Die Bundesrepublik trage eine besondere Verantwortung für das europäische Projekt, so Steinmeier. Im Zentrum des Kontinents dürfe daher ,kein ängstliches Herz schlagen“. Welche Taten damit genau gemeint sind, buchstabierten Bundespräsident, Verteidigungsministerin und Außenminister jedoch nicht genauer aus.

\section{Eine Neuorientierung des Westens?}

Steht die „Ideenlosigkeit“ (Riecke 2020) im deutschen außen- und sicherheitspolitischen Diskurs also exemplarisch dafür, dass der Westen auf der Suche nach seiner 
Identität weiter auf der Stelle tritt? Entgegen gelegentlicher Rufe, die Debatten in München würden sich teils in unproduktiver westlicher Nostalgie oder Selbstgeißelung verlieren (Dempsey 2019), brachte die Diskussion rund um Westlessness viele ,interessante Kontroversen“ und „,neuen Stoff zum Nachdenken“ hervor (Narlikar 2020). Beachtenswert war zudem, dass dies nicht zuletzt Wortbeiträgen aus Demokratien außerhalb des transatlantischen Westens, vor allem aus Asien, zu verdanken war. Vielleicht nur folgerichtig, da mit China die größte externe Herausforderung an die liberale internationale Ordnung dort Gestalt annimmt. Doch: Gelingt es den Verfechtern dieser Ordnung rund um die Welt eine Strategie für die sich anbahnende Ära der Großmächtekonfrontation zu entwickeln? Wie genau kann eine neue Allianz liberaler Demokratien aussehen, die anstelle des bisherigen westlichen Bündnisses eine weltpolitische Gestaltungsrolle ausübt? Dies bleiben einige der unbeantworteten Fragen, die es auf Münchner Sicherheitskonferenzen der kommenden Jahre weiter zu diskutieren gilt. Dementsprechend steht das transatlantische Bündnis vor der Herausforderung, das eigene Wertefundament zu stärken und sich zugleich in einer neuen Ordnung zu orientieren, die zunehmend von anderen Mächten - und potenziellen Verbündeten - mitgestaltet wird.

\section{Literatur}

Bialecki, M., Mölling, C., \& Schwarzer, D. (2020, 17. Feb.). Europa muss Führungsanspruch zeigen. DGAP Kommentar, 1. Deutsche Gesellschaft für Auswärtige Politik. https://dgap.org/de/forschung/ publikationen/europa-muss-fuehrungsanspruch-zeigen. Zugegriffen: 31. März 2020.

BMVg - Bundesministerium der Verteidigung. (2020, 14. Feb.). Münchner Sicherheitskonferenz: Kampf gegen IS geht weiter. https:/www.bmvg.de/de/aktuelles/muenchner-sicherheitskonferenz-2020kampf-gegen-is-geht-weiter-182664. Zugegriffen: 31. März 2020.

Borrell Fontelles, J. (2019, 07. Okt.). Hearing of Josep Borrell Fontelles, High Representative/Vice President-designate of the European Commission: Opening statement by Josep Borrell Fontelles. European Parliament. https://multimedia.europarl.europa.eu/en/hearing-of-josep-borrell-fontelles-highrepresentative-vice-president-designate-of-the-european-commission-opening-statement_I178140V_v. Zugegriffen: 31. März 2020.

Brössler, D. (2020, 16. Feb.). Europa im Zangengriff. Sueddeutsche.de. https://www.sueddeutsche.de/ politik/muenchen-sicherheitskonferenz-eu-trump-1.4799980. Zugegriffen: 31. März 2020.

Bunde, T. (2014). Deutsche Verantwortung, transatlantische Verstimmung und syrische Verzweiflung. Ein Bericht über die 50. Münchner Sicherheitskonferenz. Zeitschrift für Außen- und Sicherheitspolitik, 7(2), 237-250.

Bunde, T., Carr, R., Eisentraut, S., Erber, C., Hammelehle, J., Hartmann, L., Kabus, J., Stärk, F., \& Voje, J. (2020, 11. Feb.). Munich Security Report 2020 - Westlessness. https://securityconference.org/ publikationen/munich-security-report-2020. Zugegriffen: 31. März 2020.

Casdorff, S.-A. (2020, 17. Feb.). Willkommen in der Welt der Macht. Der Tagesspiegel. https:// www.tagesspiegel.de/politik/muenchner-sicherheitskonferenz-willkommen-in-der-welt-der-macht/ 25554430.html. Zugegriffen: 31. März 2020.

Cohen, R. (2019, 15. Feb.). Munich or a requiem for the West. The New York Times. https://www.nytimes. com/2019/02/15/opinion/munich-trump-europe-iran.html. Zugegriffen: 31. März 2020.

De Waal, T. (2020, 25. Feb.). Time for an Armenia-Azerbaijan history ceasefire. Carnegie Europe. https:// carnegieeurope.eu/strategiceurope/81137. Zugegriffen: 31. März 2020.

Dempsey, J. (2019, 17. Feb.). Misplaced nostalgia for the old West. Carnegie Europe. https://carnegieeurope. eu/strategiceurope/78384. Zugegriffen: 31. März 2020.

Drezner, D. (2020, 17. Feb.). What I learned at the 2020 Munich Security Conference. The Washington Post. https://www.washingtonpost.com/outlook/2020/02/17/what-i-learned-2020-munich-securityconference/. Zugegriffen: 31. März 2020.

Fontaine, R. (2020, 17. Feb). Power and pretzels in Munich, 2020. War on the Rocks. https://warontherocks. com/2020/02/power-and-pretzels-in-munich-2020/. Zugegriffen: 31. März 2020. 
hr2-kultur. (2020, 17. Feb.). „Westlessness“ - Die Welt sortiert sich neu. https://www.hr2.de/podcasts/dertag/westlessness--die-welt-sortiert-sich-neu,podcast-episode-64970.html. Zugegriffen: 31. März 2020.

Joffe, J. (2020, 19. Feb). So schlecht geht es dem Westen nicht. https://www.zeit.de/politik/2020-02/ muenchner-sicherheitskonferenz-westlessness-demokratie-europa. Zeit Online. Zugegriffen: 31. März 2020.

Kaim, M., \& Schulz, R. (2020). The EU will not be able to enforce the UN arms embargo in Libya. SWP Comment, 8. Stiftung Wissenschaft und Politik. https://www.swp-berlin.org/en/publication/the-euwill-not-be-able-to-enforce-the-un-arms-embargo-in-libya/. Zugegriffen: 31. März 2020.

Narlikar, A. (2020). Musik von der MuSiKo. Tagesspiegel Causa. https://causa.tagesspiegel.de/kolumnen/ amrita-narlikar-1/musik-von-der-musiko-2020.html. Zugegriffen: 31. März 2020.

Présidence de la République. (2020, 07. Feb.). Speech of the President of the Republic on the defense and deterrence strategy. https://www.elysee.fr/emmanuel-macron/2020/02/07/speech-of-the-presidentof-the-republic-on-the-defense-and-deterrence-strategy.en. Zugegriffen: 31. März 2020.

Riecke, T. (2020, 16. Feb.). Europas Sprach- und Ideenlosigkeit ist die traurige Bilanz der Münchner Sicherheitskonferenz. Handelsblatt. https://www.handelsblatt.com/meinung/kommentare/kommentareuropas-sprach-und-ideenlosigkeit-ist-die-traurige-bilanz-der-muenchner-sicherheitskonferenz/ 25543860.html. Zugegriffen: 31. März 2020.

Rinke, A. (2020, 17. Feb.). Vorbild Airbus - Grünen-Chefin will europäisches 5G-Konsortium. Reuters. https://de.reuters.com/article/deutschland-5g-gr-ne-idDEKBN20B0XZ. Zugegriffen: 31. März 2020.

Russell, M. (2018). EU sanctions: A key foreign and security policy instrument. European Parliamentary Research Service. https://www.europarl.europa.eu/RegData/etudes/BRIE/2018/621870/EPRS_ BRI(2018)621870_EN.pdf. Zugegriffen: 31. März 2020.

Sanger, D., \& Erlanger, S. (2020, 15. Feb.). 'The West is winning,' Pompeo said. The West wasn't buying it. The New York Times. https://www.nytimes.com/2020/02/15/world/europe/pompeo-munichconference.html. Zugegriffen: 31. März 2020.

zdf.de. (2020, 16. Feb.). Münchner Sicherheitskonferenz: „Europa muss zu Interventionen bereit sein“. https://www.zdf.de/nachrichten/politik/aussenministertreffen-sicherheitskonferenz-libyen-100.html. Zugegriffen: 31. März 2020. 